\title{
Tumores virilizantes del ovario: presentación de dos casos manejados mediante cirugía por monopuerto
}

\author{
Alexandra Madariaga L. ${ }^{1}$, Marife Araujo Q. ${ }^{2}$, Mónica Gil M. ${ }^{3}$, Gabriel Rendón P.4, \\ René Pareja F. \\ ${ }^{1}$ Residente Ginecología Oncológica, Hospital de San José, Bogotá, Colombia. ${ }^{2}$ Ginecóloga Centro Médico La Trinidad, \\ Caracas, Venezuela. ${ }^{3}$ Patóloga, Clínica Las Américas, Medellín, Colombia. ${ }^{4}$ Ginecólogo, Instituto de Cancerología, Las \\ Américas, Medellín, Colombia.
}

\section{RESUMEN}

Los tumores virilizantes, corresponden al $1 \%$ de todos los tumores funcionales del ovario. Estos tipos de tumores virilizantes se originan de las células pluri-potenciales del estroma ovárico, tienen la capacidad de secretar 17-hidroxiprogesterona, testosterona y androstenediona, desencadenando hiperandrogenismo clínico. Son catalogados como de bajo potencial maligno, con un patrón de crecimiento lento, bien diferenciados, diagnosticados en su mayoría en estadío I y II, de buen pronóstico y típicos de mujeres en edad reproductiva. El objetivo de esta comunicación es presentar dos casos clínicos con diagnóstico de tumor virilizante de ovario, tratadas con cirugía laparoscópica por mono puerto.

\section{PALABRAS CLAVE: Tumores virilizantes del ovario, tumores de Sertoli-Leydig, laparoscopia monopuerto}

\section{SUMMARY}

Virilizing tumors, corresponding to $1 \%$ of all functional ovarian tumors. Those type of virilizing tumors originate from pluripotential ovarian stromal cells and have the capacity to secrete 17-hydroxyprogesterone, testosterone and androstenedione, triggering clinical hyperandrogenism. They are classified as low malignant potential, well differentiated, with a pattern of slow growth, mostly diagnosed in stage I and II, with good prognosis and typical of women of reproductive age. The aim of this paper is to present two cases of virilizing ovarian tumor treated by mono port laparoscopic surgery.

\section{KEY WORDS: Virilizing ovarian tumors, Sertoli-Leydig ovarian tumors, monoport laparoscopy}

\section{INTRODUCCIÓN}

Los tumores estromales y de los cordones sexuales, corresponden al $7 \%$ de las neoplasias ováricas malignas, mientras que los tumores de Sertoli-Leydig y los tumores de las células de Leydig representan menos del $0,5 \%$ y del $0,1 \%$ de todos los tumores ováricos, respectivamente (1). Estos tipos de tumores virilizantes se originan de las células pluri-potenciales del estroma ovárico, tienen la capacidad de secretar 17-hidroxiprogesterona, testosterona y androstenediona, desencadenando hiperandrogenismo, con manifestaciones clínicas características. Los tumores de Sertoli-Leydig causan el $40 \%$ de la virilización clínica (2), sin embargo esto no debe considerarse como una manifestación obligatoria, y en la mayoría de los casos, la amenorrea secundaria llega a ser el único signo clínico (3).

Se considera para este tipo de tumores el 
manejo quirúrgico como tratamiento estándar, con resolución de la sintomatología y normalización de perfil hormonal. Dadas las características sólidas del tumor, la laparotomía ha sido difundida como abordaje quirúrgico inicial, tanto para la estadificación quirúrgica completa, como para la cirugía preservadora de la fertilidad (4). Sin embargo la laparoscopia aparece como un método menos invasivo, con recuperación más rápida, sin un riesgo mayor de complicaciones respecto a la laparotomía, y sin cambios en el pronóstico oncológico (4), razón por la cual documentamos dos casos clínicos de pacientes con tumores virilizantes a las que se les realizó tratamiento quirúrgico por laparoscopia.

Este proyecto fue aprobado por el comité de ética médica e investigación del IDC las Américas, en Medellín, Colombia, previa obtención del consentimiento informado de las pacientes.

\section{Caso clínico 1}

Paciente de 44 años, Gesta 1 Para 1, remitida por cuadro clínico de 5 años de evolución, caracterizado por amenorrea, acné, hirsutismo, aumento según la paciente del tamaño del clítoris y agravamiento de la voz. Hipertensa controlada, sin otro tipo de antecedente importante. Al examen físico de ingreso, se evidencia hirsutismo cuantificado en $33 / 36$, de acuerdo a la escala de Ferriman-Gallway, observándose una distribución homogénea del vello, en cara, pecho, abdomen superior e inferior, muslos y piernas. Además se observa clítoris aumentado de tamaño y acantosis nigricans en la parte posterior del cuello, axilas y genitales. El resto de examen ginecológico fue catalogado como normal.

La ecografía transvaginal reporta una lesión sólida en el ovario derecho de $20 \times 18 \times 20 \mathrm{~mm}$; la paciente trae resultado de alfa feto proteína (AFP) y CA 125 negativos. Se solicita un perfil hormonal como estudio complementario, encontrándose testosterona total mayor a $15 \mathrm{nmol} / \mathrm{L}$ y testosterona libre mayor a $65 \mathrm{pmol} / \mathrm{l}$ (valores de referencia: testosterona total: $0,21-2,98 \mathrm{nmol} / \mathrm{L}$, testosterona libre: 10,4-65,9 pmol/L)

De acuerdo a los resultados de las mediciones hormonales y el síndrome hiperandrogénico al examen físico, se planteó un diagnóstico de tumor virilizante de ovario derecho. Se propuso a la paciente realizar una laparoscopia con resección de tumor de ovario y biopsia por congelación. Durante el acto operatorio, se realizó salpingo-ooforectomía derecha por monopuerto, con extracción de la pieza quirúrgica mediante el uso de un separador abdominal elástico, tipo Alexis ${ }^{\circledR}$ Protector, Applied Medical. Adicionalmente se toman lavados pélvicos y se inspecciona toda la cavidad sin evidencia de enfermedad macroscópica extra ovárica. La biopsia por congelación reporta un tumor de Leydig.
El estudio anatomopatológico final, confirma el diagnóstico de un tumor de células de Leydig de bajo potencial maligno (Figuras 1 y 2). La citología del lavado peritoneal fue negativa, por lo que se clasifica como un estadío IA. No se presentaron complicaciones en el posoperatorio. A los dos meses del procedimiento inicial, durante su segundo control se observa reducción de la acantosis en el cuello, recuperación de su tono de voz y normalización de los niveles séricos de andrógenos (Tabla I). Luego de cinco años de seguimiento no se ha documentado recurrencia de su enfermedad.

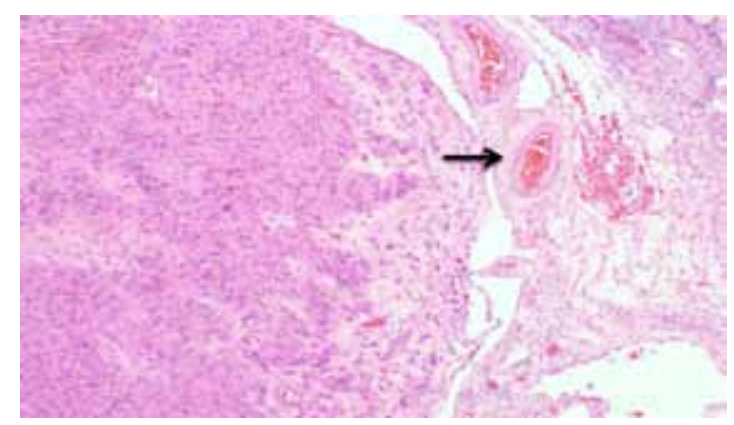

Figura 1. Tumor de células de Leydig. Caso 1. HE, 20x.

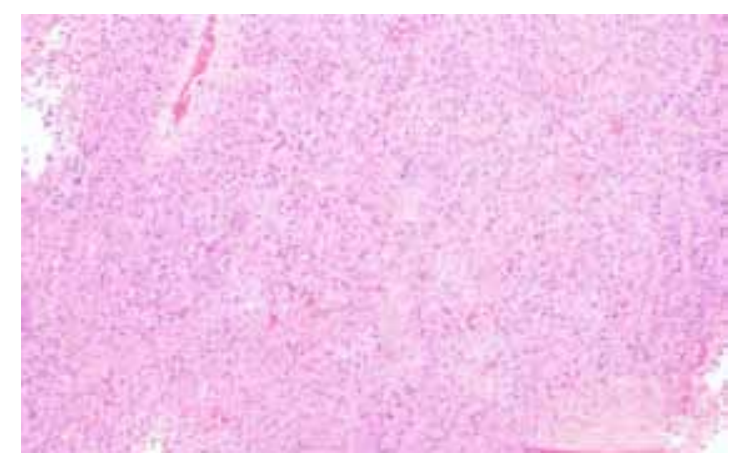

Figura 2. Tumor de células de Leydig. Caso 1. HE, 40x.

\section{Tabla I \\ PERFIL HORMONAL PRE Y POSQUIRÚRGICO. CASO 1}

\begin{tabular}{lcc}
\hline Hormonas & $\begin{array}{c}\text { Valores } \\
\text { prequirúrgico }\end{array}$ & $\begin{array}{c}\text { Valores } \\
\text { posquirúrgico }\end{array}$ \\
\hline Testosterona total & $>15 \mathrm{nmol} / \mathrm{L}$ & $0,23 \mathrm{nmol} / \mathrm{L}$ \\
Testosterona libre & $>65 \mathrm{pmol} / \mathrm{L}$ & $0,73 \mathrm{pmol} / \mathrm{L}$ \\
$\mathrm{FSH}$ & $0,35 \mathrm{UI} / \mathrm{L}$ & $5,27 \mathrm{UI} / \mathrm{L}$ \\
$\mathrm{LH}$ & $0 \mathrm{UI} / \mathrm{L}$ & $70,3 \mathrm{UI} / \mathrm{L}$ \\
\hline
\end{tabular}




\section{Caso clínico 2}

Paciente de 31 años, Gesta 1 Para 1, con cuadro clínico de un año de evolución caracterizado por oligomenorrea asociada a la aparición de acné, hirsutismo, aumento de la masa muscular y agravamiento de la voz. Sin ningún otro tipo de patología o antecedente de importancia. Durante el examen físico inicial, llama la atención lo grueso de su voz, hirsutismo por la escala de Ferriman-Gallway cuantificado en $28 / 36$, distribuido en cara, pecho, abdomen inferior, muslos y piernas. Adicionalmente presenta acantosis nigricans en la parte posterior del cuello, axilas y genitales, de reciente aparición (Figura 3). Al examen pélvico no se identifican masas ni alteraciones de importancia, pero se aprecia un clítoris ligeramente aumentado de tamaño.

La ecografía pélvica transvaginal, evidencia ovarios de aspecto poliquístico, sin embargo la tomografía axial computarizada (TAC) con contraste, visualizó dentro del ovario derecho, una lesión sólida, de $30 \times 30 \mathrm{~mm}$, redondeada, con bordes bien delimitados y sin signos de infiltración a otras estructuras. El perfil hormonal reporta testosterona total $285 \mathrm{nmol} / \mathrm{L}$ y testosterona libre $22,38 \mathrm{nmol} / \mathrm{L}$.

Con diagnóstico de tumor virilizante de ovario izquierdo, la paciente es llevada a laparoscopia con biopsia por congelación, la cual es reportada como tumor de Sertoli-Leydig. Se realiza salpingo-ooforectomía izquierda por monopuerto mediante el uso de un separador abdominal elástico, tipo Alexis $₫$ Protector, Applied Medical.

El estudio anatomopatológico definitivo, confirmó el diagnóstico de un tumor de células de SertoliLeydig bien diferenciado, sin elementos heterólogos ni patrón retiforme (Figuras 4, 5 y 6). En su segundo mes posoperatorio, se observó una disminución del área de acantosis tanto en nuca como axilas, normalización del ciclo y del perfil hormonal (Tabla II).

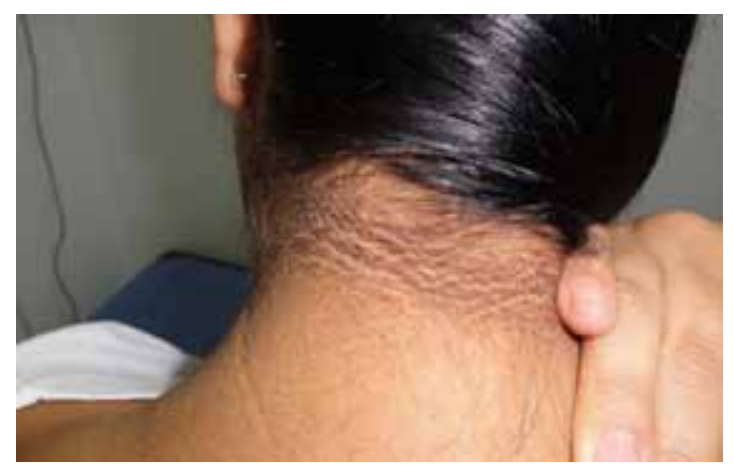

Figura 3. Acantosis nigricans en la nuca. Caso clínico 2.

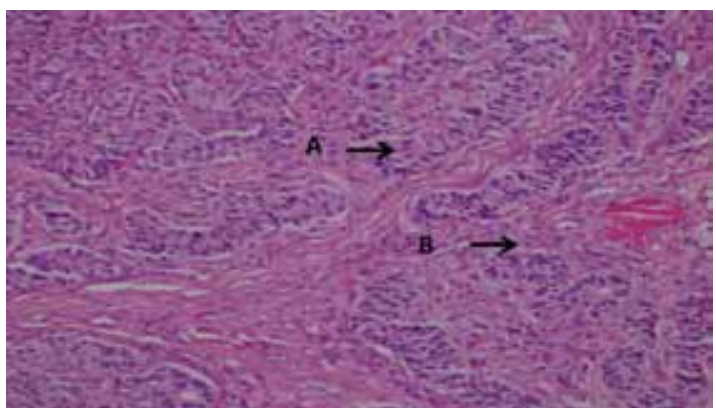

Figura 4. A. Componente tubular de células de Sertoli. B. Componente celular de Leydig en intersticio. Caso 2. HE, 20x.

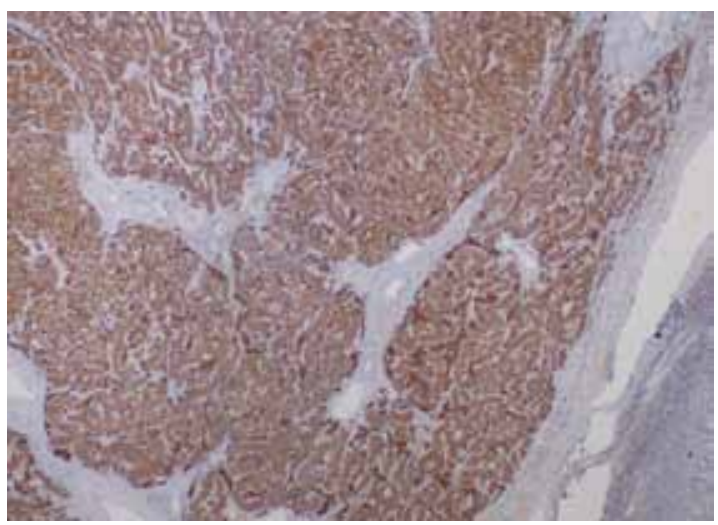

Figura 5. Inhibina intensamente positiva. Caso 2. Inmunohistoquímica, 20x.

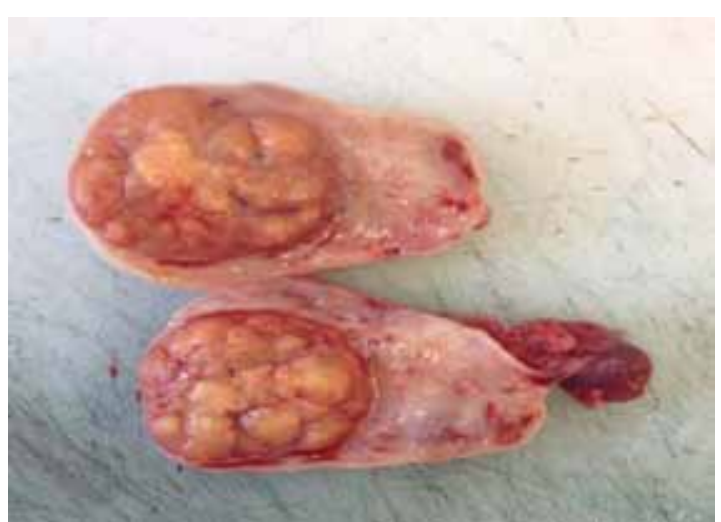

Figura 6. Ovario izquierdo de $5 \times 3,5 \times 3 \mathrm{~cm}$, superficie lisa, vascularizada. Masa bien delimitada al corte, amarilla, de $2,5 \times 2,1 \mathrm{~cm}$, rodeada por parénquima ovárico de aspecto usual. Caso 2. 


\section{Tabla II \\ PERFIL HORMONAL PRE Y POSQUIRÚRGICO. CASO 2}

\begin{tabular}{lcc}
\hline Hormonas & $\begin{array}{c}\text { Valores } \\
\text { prequirúrgico }\end{array}$ & $\begin{array}{c}\text { Valores } \\
\text { posquirúrgico }\end{array}$ \\
\hline Testosterona total & $285 \mathrm{nmol} / \mathrm{L}$ & $22,38 \mathrm{nmol} / \mathrm{L}$ \\
Testosterona libre & $93 \mathrm{nmol} / \mathrm{L}$ & $0,5 \mathrm{pmol} / \mathrm{L}$ \\
FSH & $5,99 \mathrm{UI} / \mathrm{L}$ & $6,75 \mathrm{UI} / \mathrm{L}$ \\
$\mathrm{LH}$ & $3,64 \mathrm{UI} / \mathrm{L}$ & $2,99 \mathrm{UI} / \mathrm{L}$ \\
\hline
\end{tabular}

\section{DISCUSIÓN}

Los tumores virilizantes, corresponden al $1 \%$ de todos los tumores funcionales del ovario (5). Se caracterizan por la presencia de estructuras testiculares productoras de andrógenos (6). El hiperandrogenismo inducido, se convierte en la principal causa de disfunción ovárica, hemorragia uterina anormal, amenorrea secundaria y síndromes virilizantes caracterizados por hirsutismo, alopecia, acné, clitoromegalia, aumento de la libido, cambios en la distribución de la grasa corporal, aumento de la masa muscular, atrofia mamaria y agravamiento de la voz (7).

Entre los tumores virilizantes más comunes se encuentran los de Sertoli-Leydig y los de las células de Leydig, haciendo parte de los tumores estromales de los cordones sexuales y de tumores de células esteroideas, respectivamente $(5,8)$. Son catalogados como de bajo potencial maligno, con un patrón de crecimiento lento, bien diferenciados, diagnosticados en su mayoría en estadío I y II, de buen pronóstico y típicos de mujeres en edad reproductiva, con edad media de 28 años (2). Adicionalmente, los síntomas y los hallazgos clínicos propios de estos tumores, son de inicio gradual, presentándose 5 a 7 años, antes del diagnóstico final (7).

Los tumores de Sertoli-Leydig pueden ser bien diferenciados, moderada y pobremente diferenciados, con patrón retiforme o con elementos heterólogos $(3,9)$. Los bien diferenciados, se consideran benignos y sin riesgo de recurrencia, con una supervivencia global a 5 años del $100 \%$. Cerca del $11 \%$ de los tumores con diferenciación intermedia y el $59 \%$ de los pobremente diferenciados son malignos, con tasas de supervivencia a 5 años del $87 \%$ y $41 \%$ respectivamente $(3,10)$.

Los tumores virilizantes, tiene un perfil hormonal clásico, con valores elevados de testosterona en el $80 \%$ de los casos, en asociación con aumento en los niveles de 17-hidroxiprogesterona y androstenediona, pero con niveles normales de esteroides adrenales, como cortisol y sulfato de dehidroepiandrosterona (7). La resección quirúrgica de estos tumores, mejora significativamente los signos de hiperandrogenismo durante el posoperatorio (1), evidenciándose una regresión a valores normales de las hormonas a los 8-10 días de la cirugía (3).

Ecográficamente, se caracterizan por ser pequeños nódulos con diámetros promedio de $3 \mathrm{~cm}$, sólidos, no calcificados, $95 \%$ unilaterales, no asociados a ascitis $(3,7,11)$. La tomografía axial computada (TAC) no presenta una elevada sensibilidad, sin embargo la evidencia de un aumento en el volumen ovárico puede llamar la atención (7).

Poco se conoce acerca del compromiso ganglionar en los pacientes con tumores estromales y de los cordones sexuales. Brown y cols (12), en su estudio retrospectivo determina el riesgo de metástasis linfática en 58 pacientes con tumores de Sertoli-Leydig llevadas a estadificación quirúrgica, sin encontrar ningún ganglio positivo. Igualmente Thrall y cols (8), no logran demostrar compromiso ganglionar entre 47 pacientes con tumor de SertoliLeydig, sometidas a linfadenectomía pélvica y para aórtica, que en términos de factores clínicos asociados a la supervivencia, plantean el tamaño tumoral como el principal predictor de supervivencia, sin evidencia de recurrencia en mujeres con lesiones menores a $7 \mathrm{~cm}$.

El tratamiento primario estándar en este tipo de tumores, continúa siendo la cirugía. En una revisión retrospectiva llevada a cabo en Taiwán, en un periodo de 20 años se identificaron 23 pacientes con tumores de Sertoli-Leydig (2), donde se plantea la cistectomía o salpingo-ooforectomía unilateral ante deseo de fertilidad. También se ha planteado la histerectomía abdominal total con salpingo-ooforectomía bilateral ante la paridad satisfecha (6). En cualquiera de los dos escenarios, para lograr completar la estadificación quirúrgica, se debe realizar una exploración pélvica y abdominal, lavados, biopsias peritoneales, omentectomía y resección de cualquier otro tipo de lesión sospechosa (6).

La laparoscopia como abordaje quirúrgico usual en el manejo de este tipo de tumores no se ha logrado popularizar dada la naturaleza sólida de los mismos. Sin embargo, debe considerarse como la técnica más adecuada, al ser menos invasiva, con mejores resultados estéticos y recuperación rápida (6).

Shim y cols (4), reportan su experiencia de manejo laparoscópico en 28 pacientes con tumores no epiteliales de ovario, evaluando la posibilidad y seguridad de este abordaje. Describe la necesidad de una evaluación sistemática de la cavidad, con extracción del tumor a través del puerto de 10 $\mathrm{mm}$ con ayuda de endo-bolsas, y en solo un caso mano asistida dado el tamaño de la lesión. Se 
describe como de $6,5 \mathrm{~cm}$ el tamaño promedio de las lesiones extraídas por laparoscopia con ruptura intraoperatoria del $17,9 \%$. El tiempo promedio de cirugía fue 102 minutos, con un sangrado promedio de $100 \mathrm{cc}$, sin complicaciones intraoperatorias ni necesidad de laparotomía. El seguimiento medio que se le dio a estos paciente fue de 34,5 meses, sin muerte por la enfermedad y una sola recaída a cerebro y pulmón a los ocho meses del tratamiento.

La terapia adyuvante, no ha demostrado beneficio en la práctica clínica. No se ha logrado mostrar una diferencia significativa en términos de supervivencia libre de enfermedad o supervivencia global, entre los pacientes que reciben o no adyuvancia, y el régimen óptimo aún permanece poco claro. Sin embargo, parece razonable el uso de quimioterapia basada en platino ante tumores metastásicos, de diferenciación intermedia, pobremente diferenciados o con patrón retiforme $(3,9)$.

Las dos pacientes de este reporte, presentaron síndrome hiperandrogénico, de progresión lenta, y alteraciones del perfil hormonal, con elevación de los niveles de testosterona total y libre, e imágenes de lesiones anexiales sólidas, menores a $5 \mathrm{~cm}$. Ambas fueron tratadas con cirugía mínimamente invasiva, por monopuerto, con exploración completa de la cavidad y sin identificación de enfermedad extra ovárica. Los hallazgos histopatológicos son compatibles con tumores virilizantes bien diferenciados diagnosticados en estadíos tempranos, sin componente heterólogo ni retiforme, y sin indicación de terapia adyuvante. Durante el seguimiento hubo normalización de los niveles hormonales y desaparición progresiva de los signos clínicos de virilización. Hasta el momento ambas pacientes se encuentran vivas y libres de enfermedad.

\section{CONCLUSIÓN}

Se plantea la cirugía laparoscópica por monopuerto como alternativa de elección para el tratamiento de tumores virilizantes de ovario, como técnica menos invasiva, con mejores resultados estéticos, tiempos más cortos de recuperación y sin alteración del pronóstico oncológico.

\section{REFERENCIAS}

1. Nardo LG, Ray DW, Laing I, Williams C, McVey RJ, Seif MW. Ovarian Leydig cell tumor in a peri-menopausal woman with severe hyperandrogenism and virilization. Gynecol Endocrinol 2005;21(4):238-41.

2. Weng CS, Chen MY, Wang TY, Tsai HW, Hung YC, Yu KJ, et al. Sertoli-Leydig cell tumors of the ovary: a Taiwanese Gynecologic Oncology Group study. Taiwan J Obstet Gynecol 2013;52(1):66-70.

3. Gui T, Cao D, Shen K, Yang J, Zhang Y, Yu Q, et al. A clinicopathological analysis of 40 cases of ovarian Sertoli-Leydig cell tumors. Gynecol Oncol 2012;127(2):384-9.

4. Shim SH, Kim DY, Lee SW, Park JY, Kim JH, Kim YM, et al. Laparoscopic management of early-stage malignant nonepithelial ovarian tumors: surgical and survival outcomes. Int J Gynecol Cancer 2013;23(2):24955.

5. Persechini ML, Motton S, Leguevaque $P$, Donadille $F$, Escourrou G, Vierasu B, et al. Virilising ovarian tumour: a case associating a Sertoli-Leydig cell tumour and a Brenner tumour. Gynecol Endocrinol 2011;27(5):345-50.

6. Sigismondi C, Gadducci A, Lorusso D, Candiani M, Breda E, Raspagliesi F, et al. Ovarian Sertoli-Leydig cell tumors. a retrospective MITO study. Gynecol Oncol 2012;125(3):673-6.

7. Juniarto $A Z$, Setiawati BA, Ediati A, van der Zwan YG, Looijenga $\mathrm{LH}$, de Jong $\mathrm{FH}$, et al.Virilization due to androgen hypersecretion in a patient with ovarian leydig cell tumor: diagnostic and psychosocial implications. Acta Med Indones 2013;45(2):130-5.

8. Thrall MM, Paley P, Pizer E, Garcia R, Goff BA. Patterns of spread and recurrence of sex cord-stromal tumors of the ovary. Gynecol Oncol 2011;122(2):242-5.

9. Grove A, Vestergaard V. Ovarian Sertoli-Leydig cell tumor of intermediate grade with heterologous elements of rhabdomyosarcoma. A case report and a review of the literature. Ann Diagn Pathol 2006;10(5):288-93.

10. Young RH, Scully RE. Ovarian Sertoli-Leydig cell tumors. A clinicopathological analysis of 207 cases. Am J Surg Pathol 1985;9(8):543-69.

11. Outwater EK, Marchetto B, Wagner BJ. Virilizing tumors of the ovary: imaging features. Ultrasound Obstet Gynecol 2000;15(5):365-71.

12. Brown J, Sood AK, Deavers MT, Milojevic L, Gershenson DM. Patterns of metastasis in sex cord-stromal tumors of the ovary: can routine staging lymphadenectomy be omitted? Gynecol Oncol 2009;113(1):8690 . 